\title{
Construção de competências do enfermeiro para implantar unidade de terapia intensiva neonatal cardiológica
}

RESUMO | Objetivo: Construir as competências profissionais necessárias do enfermeiro na implementação de UTI Neonatal Cardiológica. Método: Revisão integrativa, utilizando os descritores Unidade de Terapia Intensiva Neonatal; Pediátrica; Infantil; Cardiologia; Enfermeiro; Papel do Enfermeiro; Competências Profissionais, nas bases Medline, LiLaCS e BDENF. Incluiu artigos primários, de revisão, na integra, idiomas inglês, português, espanhol e francês, excluindo duplicadas e não relacionados ao tema. Resultado: foram considerados subsídios de acordo com os artigos selecionados dos quais onze competências foram construídas: conhecer a missão, visão, valores institucionais; definir o perfil de paciente; participar da organização da estrutura física; composição da equipe multiprofissional; prever serviços, prover materiais e equipamentos; gerenciar custos; estabelecer atendimento ao paciente e família; construir indicadores; estruturar unidade com programas de segurança ao paciente; criar protocolos assistenciais. Conclusão: Este estudo contribui para a prática do enfermeiro com subsídios para a implementação da UTI Neonatal Cardiológica por meio de recursos e estratégias de gestão.

Palavras-chaves: Enfermagem; Unidade de Terapia Intensiva Neonatal; Competência Profissional.

ABSTRACT | Objective: To build the necessary professional competences of the nurse in the implementation of a Cardiac Neonatal ICU. Method: This is an Integrative Review study, using the descriptors Neonatal Intensive Care Unit; Pediatric; Child; Cardiology; Nurse; Nurse's Role; Professional Competencies, in Medline, LiLaCS and BDENF databases. It included primary and review studies that were available in their full version, in English, Portuguese, Spanish and French, excluding duplicates and articles unrelated to the topic. Results: subsidies were considered according to the selected articles, of which eleven competencies were built: to know the mission, vision, institutional values; to define the patient profile; to participate in the organization of the physical structure; composition of the multidisciplinary team; to predict services, provide materials and equipment; to manage costs; to establish patient and family care; to build indicators; to structure a unit with patient safety programs; to create assistance protocols. Conclusion: This study contributes to the practice of the nurse with subsidies for the implementation of a Cardiac Neonatal ICU through resources and management strategies.

Keywords: Nursing; Neonatal Intensive Care Units; Professional Competence.

RESUMEN | Objetivo: Desarrollar las habilidades profesionales necesarias del enfermero en la implementación de UCI Cardiológicas Neonatales. Método: Revisión integradora, usando los descriptores Unidad de Cuidados Intensivos Neonatales; Pediátrico; Infantil; Cardiología; Enfermero; Papel del enfermero; Habilidades profesionales, basadas en Medline, LILACS y BDENF. Incluyo artículos primarios, de revisión, completos, idiomas inglés, portugués, español y francés, excluyendo duplicados y no relacionados con el tema. Resultado: los subsidios se consideraron de acuerdo con los artículos seleccionados a partir de los cuales se construyeron once competencias: conocer la misión, la visión, los valores institucionales; definir el perfil del paciente; participar en la organización de la estructura física; composición del equipo multidisciplinario; predecir servicios, proporcionar materiales y equipos; gestionar los costos; establecer atención a pacientes y familiares; construir indicadores; estructurar una unidad con programas de seguridad al paciente; crear protocolos de asistencia. Conclusión: Este estudio contribuye a la práctica del enfermero con subsidios para la implementación de la UCI Cardiológica Neonatal a través de recursos y estrategias de gestión.

Palabras claves: Enfermería; Unidad de Cuidados Intensivos Neonatales; habilidad profesional.

\section{Vitor Latorre Souza}

Enfermeiro Residente em Enfermagem Cardiovascular do Instituto Dante Pazzanese de Cardiologia. Graduado na Faculdade de Medicina de Marília - FAMEMA.

\section{Dra Rika Miyahara Kobayashi}

Diretora do Serviço de Educação Continuada, Coordenadora da Comissão de Residência Multiprofissional (COREMU) e do Programa de Residência Multiprofissional em Saúde Cardiovascular do Instituto Dante Pazzanese de Cardiologia. Doutora em Enfermagem pela Escola de Enfermagem da USP.

\section{$\mathrm{Dr}^{\circ}$ Sérgio Henrique Simonetti}

Chefia de Enfermagem de Unidade de Exames Cardiológicos não Invasivos. Pós-Doutorado em Ciências pelo IDPC-USP. Doutor e Mestre em Ciências pela EEUSP.

Recebido em: 08/01/2020 Aprovado em: 31/03/2020
INTRODUÇÃO

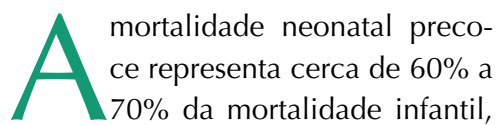
e $25 \%$ dos óbitos ocorrem no primeiro dia de vida, sendo as cardiopatias congênitas responsáveis por cerca de 10\% dos óbitos infantis e $20 \%$ a $40 \%$ dos óbitos decorrentes de malformações segundo o Plano Nacional de Assistência à Criança com Cardiopatia Congênita. ${ }^{(1)}$

A cada ano nascem no Brasil cerca de 29,8 mil cardiopatas - recorte de $1 \%$ para 
dados de nascidos vivos em 2014. Uma vez que em apenas $20 \%$ dos casos a remissão é espontânea, estima-se que $80 \%$ do total (mais de 23,8 mil crianças) precisarão de intervenção cirúrgica em algum momento do seu desenvolvimento, sendo que a metade deve ser operada ainda no primeiro ano de vida. ${ }^{(2)}$

Frente a esta demanda da população neonatal cardiopata, justifica se a necessidade de criação de Unidades de Terapia Intensiva Neonatal Cardiológica para atendimento de alta complexidade em área cardiovascular, conforme o Plano Nacional de Assistência à Criança com Cardiopatia Congênita, considerando se que em levantamento de serviços de atenção especializada de alta complexidade, conforme os dados constantes no Cadastro Nacional dos Estabelecimentos de Saúde do Brasil CNES em 2020, dentre os 328.015 hospitais gerais constantes nesse cadastrado, somente 568 eram de hospitais de alta complexidade na esfera estadual, e 1556 na esfera municipal. ${ }^{(3)}$

$\mathrm{O}$ enfermeiro pode ser um dos profissionais responsáveis pela implementação da UTI Neonatal, pois segundo a Resolução do COFEN n. 509/2016, que atualiza a norma técnica para Anotação de Responsabilidade Técnica pelo Serviço de Enfermagem e define as atribuições do enfermeiro Responsável Técnico descreve sobre a responsabilidade do enfermeiro o planejamento, organização, direção, coordenação, execução e avaliação dos serviços de Enfermagem, a quem é concedida, pelo Conselho Regional de Enfermagem.

Mediante importante atribuição do enfermeiro, verificamos nas Diretrizes Curriculares Nacionais para os Cursos de Graduação em Enfermagem em vigor, que as competências gerais a serem alcançadas pelo enfermeiro são a atenção à saúde, a tomada de decisão, a comunicação, liderança, administração/gerenciamento e educação permanente. ${ }^{(4)}$

Com um olhar focado na competência de gestão, segundo um estudo sobre a temática, os recém formados manifestam ter
6

suficientes conhecimentos, consideram-se hábeis e com atitudes favoráveis no área de gestão; em mudança os formados com maior experiência trabalhista, ainda que consideram que têm suficiente conhecimento, sua habilidade é média e reportam uma atitude desfavorável para a gestão. ${ }^{(5)}$

Neste sentido, buscou-se literaturas sobre a estruturação de um serviço de Unidade de Terapia Intensiva Neonatal Cardiológica no Brasil, e verificou se a escassez de literaturas bem como de definições de competências profissionais para implementação deste serviço.

Neste estudo, optamos por definir a competência profissional pois esse conceito envolve os aspectos cognitivos, técnicos, sociais e afetivos presentes em uma prática profissional, mostrando que competência tem a ver com o conjunto de conhecimentos, habilidades e atitudes interdependentes e necessárias à conclusão de uma determinada atribuição. Também é possível definir como "um saber agir responsável e reconhecido, que implica mobilizar, integrar, transferir conhecimentos, recursos e habilidades, que agreguem valor econômico à organização e valor social ao indivíduo". ${ }^{(6,7)}$

Assim, a realização deste estudo, justifica-se pela carência de novas estruturações de UTI Cardiológica Neonatal, necessidade de formação de competências para esta estruturação e escassez de literatura que possibilite esta construção. Desta forma, este estudo teve como objetivo, construir as competências profissionais do enfermeiro para a implementação de uma UTI Neonatal Cardiológica.

\section{MÉTODO}

Trata-se de uma revisão integrativa da literatura, realizada no período de abril a junho de 2019. Para tanto, seguiram-se seis etapas: estabelecimento da questão de pesquisa, definição dos critérios de inclusão e exclusão dos estudos, categorização dos estudos, avaliação dos estudos da revisão, interpretação dos resultados e por fim síntese do conhecimento. ${ }^{(8)}$ 
$\mathrm{Na}$ primeira etapa, elaborou-se o tema por meio da estratégia "PICO" onde o P de população (enfermeiro no contexto hospitalar); I de intervenção ou área de interesse (conhecimentos, atitudes, habilidades e práticas); C de comparação entre intervenção ou grupo (não foi empregado) e o O de desfecho (as competências profissionais necessárias) e a pergunta de pesquisa: "Quais as competências pro- fissionais necessárias do enfermeiro para implementar uma Unidade de Terapia Intensiva Neonatal Cardiológica".

Utilizou-se uma biblioteca virtual internacional de saúde, a PubMed, e as seguintes bases de dados MEDLINE, LILACS e BDENF através dos descritores Professional Competence, Nurse e Nurse's Role, Neonatal Intensive Care Unit, Pediatric Intensive Care Units, Infant e Cardiology.
A inclusão do descritor Pediatric Intensive Care Units foi decorrente desta incluir artigos relacionados à neonatologia.

Estabeleceu-se na segunda etapa, os critérios de inclusão: artigos primários e de revisão disponíveis na integra com leitura do título seguido da análise do resumo e de acesso gratuito, que especificaram quesitos pertinentes à estrutura ou organização de uma Unidade de Terapia

Tabela 1 - Distribuição de artigos identificados na pesquisa, segundo título, periódico, ano, idioma, autor, tipo de estudo, nível de evidência e assunto. São Paulo,SP, Brasil 2019.

\section{TÍTULO/PERIÓDICO}

Informing Leadership Models: Nursing and Organizational Characteristics of Neonatal Intensive Care Units in Freestanding Children's Hospitals. Arch Cardiovasc Dis
AUTOR DO / NÍVEL DE EVIDENCIA

\section{ASSUNTO}

Equipamentos, material, profissionais,

Toole CA; De Grazia

2018 / francês $\quad$ M;ConnorJÁ; GauHickey PA.
IV / Descritivo

estrutura, atendimento multiprofissional e abordagem familiar.

Paediatric cardiac intensive care unit: current setting and organization in 2010 . Dimens Crit Care Nurs

2010 / inglês Fraisse A; Le Bel S; Mas B; Macrae D.

VI / Descritivo e Revisão

Atribuições do enfermeiro na UTI Neonatal.

A multinational survey on the infrastructural quality of paediatric intensive care units.Annals of intensive care

2018 / Inglês Warneck, Gert, et al. VI / Descritivo

Estrutura, material, equipamentos erecursos humanos.

\section{Literature review of the impact of} nurse practitioners in critical care services.Nursing in critical care

.LaUnidad de Cardiología Pediátrica del Hospital Infantil Universitario Miguel Servet de Zaragoza. Rev. esp. pediatr.

Unidad de Cuidados Intensivos Pediátricos enel Hospital Universitario Ramón y Cajalenelaño 2015.Rev. esp. pediatr.
2011 / Inglês

FRY, M. VI / Revisão da
literatura
Assistência de Enfermagem.

\section{Jiménez ML; López}

RM; Ayerza CA; Palanca Arias, D.

Martínez JLV;

2015 / espanhol Macarrón CPC; Pérez AC; Toledo DF; Porras MS; R. Tapia Moreno.
VI / Descritivo

Missão, visão e valores, perfil dos pacientes e estrutura física.

Las Unidades de Cardiología Pediátrica y CirugíaCardiaca Infantil del Hospital Universitario y Politécnico La Fé. Rev. esp. 2014/ espanhol

Carrasco LF; Insa Jl; Boni B.

VI / Descritivo

Indicadores.

Estrutura física, profissionais, patologias, serviços prestados, segurança ao paciente, indicadores e protocolo operacional padrão. pediatr.

\section{Estudo comparativo do consumo e} gasto com medicamentos em Unidades Pediátricas de Terapia Intensiva e Semi-intensiva Rev. paul. Pediatr

\section{2 / português Zuliani, LL; Jericó, MC.}

VI / Descritivo

La Unidad de Neonatologíadel Hospital Universitario Miguel Servetenlaactualidad. Nuevosretos/Neonatology Unit of the Hospital Universitario Miguel Servet at 2015 / espanhol Gracia, SR, et al VI / Descritivo

Custos da unidade em relação material. present: new challenges

Fonte: Elaborado pelo próprio autor, 2019. 
Intensiva Neonatal ou Pediátrica, com o uso de descritos em português, inglês, espanhol e francês. Como critério de exclusão: as publicações duplicadas e artigos sem relação com o tema sobre a implantação de um serviço de Terapia Intensiva Neonatal Cardiológica.

Identificaram-se na terceira e quarta etapa os artigos pré-selecionados e selecionados, utilizando um roteiro adaptado que contemplou as bases de dados, os títulos da publicação, os periódicos, o ano de publicação, os idiomas, os objetivos, os tipos de estudo, os assuntos relacionados com o tema e o nível de evidência classificado. ${ }^{(8-9)}$

Para a análise e síntese dos estudos selecionados que são a quinta e sexta etapa utilizou-se uma tabela estruturada e construída pelos autores com a finalidade de organizar, analisar, e categorizar as seguintes informações: nome da pesquisa; nome dos autores; idioma; tipo de estudo; nível de evidência e assuntos pertinentes com a temática. Os estudos selecionados foram analisados em duplas checagens por especialistas, que após a seleção e categorização por meio da busca de conhecimentos, habilidades ou atitudes constituintes construíram as competências profissionais necessárias para a implantação de uma Unidade Intensiva Neonato Cardiológica.

\section{RESULTADOS}

Do total de 1.189 artigos, 49 estudos foram selecionados e destes nove atenderam aos critérios de elegibilidade. De nove artigos, quatro foram publicados na Espanha, um na França, três nos Estados Unidos e um no Brasil, no período entre 2010 e 2018, em revistas internacionais.

No que diz respeito ao delineamento da pesquisa, sete estudos eram qualitativos, e dois artigos quantitativos. Referente ao tipo de estudo, obtivemos sete estudos descritivos, dois de revisão narrativa. Quanto ao nível de evidência, oito estudos apresentam nível VI e um com nível IV. A tabela1 sintetiza os resultados do roteiro de coleta.
Não foram encontradas literaturas específicas sobre as competências profissionais dos enfermeiros na implementação de uma Unidade Terapia Intensiva Neonatal Cardiológicas, entretanto foram encontrados alguns conhecimentos ou habilidades necessárias em cada artigo selecionado. Assim, com a consulta da Resolução RDC n50/2002 que dispõe sobre o Regulamento Técnico para planejamento, programação, elaboração e avaliação de projetos físicos de estabelecimentos assistenciais de saúdee a Portaria $n^{\circ}$ 930/2012que define as diretrizes e objetivos para a organização da atenção integral e humanizada ao recém-nascido grave ou potencialmente grave e os critérios de classificação e habilitação de leitos de Unidade Neonatal no âmbito do Sistema Único de Saúde (SUS), obteve-se a orientação quanto à estrutura física, equipamentos e recursos humanos necessários para planejar o atendimento em uma UTI Neonatal Cardiológica. ${ }^{(11-12)}$

\section{DISCUSSÃO}

A escassez de literatura sobre implementação de unidades aponta para a necessidade do enfermeiro em se atentar para sua atribuição privativa no planejamento, organização, coordenação, execução e avaliação dos serviços da assistência de enfermagem conforme o Art. 11 da Lei $n^{\circ} 7498$ de 25 de junho de 1986, conhecida como lei do exercício profissional.

Com relação às competências e de acordo com a presente revisão, foram construídas as onze competências profissionais, a saber:

1. Conhecer a missão, visão e valores da instituição e objetivo da implantação da unidade. ${ }^{(13,22,23)}$

2. Definir o perfil de paciente atendido e epidemiologia das patologias atendidas. ${ }^{(14,24)}$

3. Participar/Conhecer da arquitetura e organização da estrutura física da unidade. ${ }^{(15,11)}$
4. Definir os profissionais de atuação do setor. ${ }^{(16,12,25)}$

5. Definir os serviços indiretos e diretos oferecidos pela unidade. ${ }^{(15,12)}$

6. Prever, prover, organizar e controlar os materiais e equipamentos disponíveis e necessários para a unidade. . $^{(17,11,26)}$

7. Estabelecer o processo de atendimento ao paciente e família. ${ }^{(18,27)}$

8. Identificar os custos financeiros de equipamentos e materiais. ${ }^{(19,26)}$

9. Construir indicadores de qualidade e resultado. ${ }^{(20,28,29)}$

10. Estruturar a unidade com programas de segurança ao paciente. ${ }^{(15,30)}$

11. Criar protocolos assistenciais, procedimentos operacionais padrão e instruções de trabalho. ${ }^{(21,31)}$

Conhecer a missão, visão, valores, da instituição da instituição é fundamental para as práticas de enfermagem e cabe ao enfermeiro gestor a propagação, construção de normas, regimentos, regulamentos, procedimentos operacionais que estejam em sintonia com a organização. Muitas instituições trabalham com programas de qualidade, certificação, divulgando-os aos seus colaboradores seja em forma de quadros, banner, na área de trabalho de seus computadores, sites ou outros meios. $^{(22-23)}$

O conhecimento do perfil dos pacientes é essencial para fundamentar o planejamento e a implementação de programas assistenciais que melhor atendam às necessidades desses cardiopatas, auxiliando na distribuição diária e na capacitação dos recursos humanos de enfermagem para o atendimento de cada paciente na Unidade de Terapia Intensiva Neonatal. ${ }^{(24)}$

Para implementar a UTI Neonatal Cardiológica no Brasil, as competências necessárias se relacionam minimamente a conhecer a RDC n ${ }^{\circ}$ 50/2002. Essa etapa é de grande importância na estruturação do projeto de criação da unidade neonatal, visto que erros nesta etapa pode comprometer o fluxo de serviço do setor. ${ }^{(11)}$ 
Ampliando o olhar de recursos humanos na orientação da estruturação do projeto de criação da UTI Neonatal Cardiológica, o enfermeiro deve ter a competência para compor e propor os profissionais de atuação, buscando a forma de melhor atender as necessidades do paciente, de maneira multiprofissional e interdisciplinar e a beira leito, e conhecendo o fluxo de atendimento. ${ }^{(12,25)}$

No que tange a gestão de recursos materiais, o enfermeiro deve ter habilidade para organizar o pleno funcionamento da unidade. O material estará presente em todas as etapas da assistência e a falta de algum item poderá comprometer diretamente algum procedimento ao qual o paciente será submetido. ${ }^{(12)}$

Geralmente o enfermeiro é responsável pelo gerenciamento de materiais,de recursos humanos, e também dos recursos financeiros, que frequentemente são escassos. Com essa realidade, torna-se muito importante o conhecimento sobre gerenciamento de custos e eficiência alocativa. ${ }^{(25)}$

$\mathrm{O}$ enfermeiro enquanto gestor também deve estabelecer o processo de atendimento ao paciente e família junto a equipe multiprofissional, sendo uma das opções, o Método Canguru que é um modelo de assistência perinatal voltado para o cuidado humanizado que reúne estratégias de intervenção biopsicossocial, cujo contato pele-a-pele, permite uma maior participação dos pais e da família nos cuidados neonatais. ${ }^{(27)}$

O uso de indicadores como ferramenta organizacional proporciona qualidade e eficácia do processo gerencial, possibilitando avaliação criteriosa, planejamento, definição de metas, seguimento e condutas de melhoria contínu$\mathrm{a}^{(28)}$.O enfermeiro gestor deve planejar o funcionamento da unidade, requerendo se conhecimento, habilidade e atitude para desempenhar tal competência ${ }^{(29)}$. Os indicadores como ferramenta organizacional mostram a necessidade do aprimoramento contínuo dos processos de enfermagem, visto que os recursos
66

humanos e tecnológicos frequentemente são renovados. ${ }^{(28)}$

$\mathrm{O}$ enfermeiro gestor deve garantir a segurança da assistência aos pacientes hospitalizados visto que eles apresentam uma situação de fragilidade e vulnerabilidade, pois não possuem o sistema imunológico completamente formado, assim a primeira barreira de proteção é imatura, associado ao fato de estarem utilizando inúmeros dispositivos invasivos, ocasionando riscos de ocorrencia de erros. A assistência a esses pacientes deve ser cercado de cuidado, atenção e qualidade no cuidado, uma vez que qualquer erro pode acarretar consequências fatais. ${ }^{(30)}$

A padronização dos procedimentos é considerada uma ferramenta gerencial moderna e tem sido amplamente discutida pela enfermagem. Os resultados do seu uso demonstram que se trata de uma estratégia que apoia a tomada de decisão por parte do enfermeiro, proporciona a oportunidade de corrigir as não conformidades, favorece que todos os profissionais prestem cuidado padronizado para o paciente seguindo os princípios técnico-científicos e, ainda, contribui para diminuir os vícios adquiridos na prática, tendo assim também finalidade educativa. ${ }^{(31)}$

Para o enfermeiro gestor da UTI Neonatal Cardiológica também é necessário que se tenha a competência de criar os protocolos e procedimentos operacionais padrão da unidade, visando à qualidade e padronização de atendimento pela equipe de trabalho. ${ }^{(31)}$

\section{CONCLUSÃO}

Os resultados enfatizaram onze competências profissionais necessárias para implantação de uma UTI neonatal cardiológica dos quais incluem o conhecer a missão, visão, valores institucionais; definir o perfil de paciente; participar da organização da estrutura física; da composição da equipe multiprofissional; prever serviços, prover materiais e equipamentos; gerenciar custos; estabelecer 
processos de atendimento ao paciente $\mathrm{e}$ família; construir indicadores; estruturar unidade com programas de segurança ao paciente; criar protocolos assistenciais e procedimentais. Essas competências construídas contribuem para a prática do enfermeiro com subsídios para a implementação da UTI Neonatal Cardiológica por meio de recursos e estratégias de gestão.

Como limitações neste estudo de revisão integrativa, verificou se escassez de literatura sobre as competências profissionais do enfermeiro na implementação de Unidade Neonatal Cardiológica, sendo necessárias sua construção a partir de conhecimentos e habilidades encontradas. Na continuidade, estudos futuros devem ser realizados com vistas à validação por expertises e clinica das competências profissionais constituídas.

\section{Referências}

1. Ministério da Saúde (BR). Portaria $\mathrm{n}^{\circ} 1.727$, de 11 de julho de 2017. Aprova o Plano Nacional de Assistência à Criança com Cardiopatia Congênita. Diário Oficial da União. 12 de julho de 2017. Seção 1:4. Disponivel em: http://bvsms. saude.gov.br/bvs/saudelegis/gm/2017/prt1727_12_07_2017.html

2. Pinto Júnior VC, Rodrigues LC, Muniz CR. Reflexões sobre a formulação de políticas de atenção cardiovascular pediátrica no Brasil. RevBrasCirCardiovasc. 2009:24(1):73-80.

3. Ministério da Saúde - Cadastro Nacional dos Estabelecimentos de Saúde do Brasil - CNES Disponível em http://tabnet.datasus.gov.br/cgi/tabcgi.exe?cnes/ cnv/atencbr.def. Acesso em 26.mar.2020.

4. Ministério da Educação (BR). Conselho Nacional de Educação. Resolução No 3, de 07 de novembro de 2001. Diretrizes curriculares nacionais do curso de graduação em Enfermagem. Diário Oficial da República Federativa do Brasil, 09 Nov 2001. Seção 1. p. 37

5. Almeida ML, Peres AM. Conhecimentos, habilidades e atitudes sobre a gestão dos formados de enfermagem de uma universidade pública brasileira. Invest Educ Enferm. 2012;30(1):66-76.

6. PERRENOUD, P.H et al.. As Competências para Ensinar no Século XXI. A Formação dos Professores e o Desafio da Avaliação. Porto Alegre : Artmed, 2002: 11-34.

7. Fleury MTL, Fleury A. Construindo o conceito de competência. Rev. adm. contemp. 2001; 5(1):183-96.

8. Mendes KS, Silveira RCCP, Galvão CM. Revisão integrativa: método de pesquisa para a incorporação de evidências na saúde e na enfermagem. Texto Contexto Enferm. Florianópolis, 2008;17(4): 758-64.

9. Ursi ES, Galvão CM. Prevenção de lesões de pele no perioperatório: revisão integrativa da literatura. Rev. Latino-Am. Enfermagem. 2006;14(1):124-31.

10. Melnyk BM, Fineout-Overholt E. Making the case for evidencebased practice. In: Melnyk BM, Fineout-Overholt E. Evidencebased practice in nursing \& healthcare. A guide to best practice. Philadelphia: Lippincot Williams \& Wilkins; 2005.3-24.

11. BRASIL. Ministério da Saúde. RDC no 50, de 21 de fevereiro de 2002. Dispõe sobre o Regulamento Técnico para planejamento, programação, elaboração e avaliação de projetos físicos de estabelecimentos assistenciais de saúde. Diário Oficial da República Federativa do Brasil. Brasília, 20 de mar. de 2002. Disponível em: http://bvsms.saude.gov.br/bvs/saudelegis/anvisa/2002/ rdc0050 21_02 2002.html

12. Ministério da Saúde (BR). Portaria $\mathrm{n}^{\circ}$ 930, de 10 de maio de 2012. Define as diretrizes e objetivos para a organização da atenção integral e humanizada ao recém-nascido grave ou potencialmente grave e os critérios de classificação e habilitação de leitos de Unidade Neonatal noâmbito do Sistema Único de Saúde (SUS). Diário Oficial da União. 11 de maio de 2012. Seção 1:4. Disponível em: http://bvsms.saude.gov.br/bvs/saudelegis/gm/2012/prt0930_10_05_2012. html

13. Gracia SR, et al. La Unidad de Neonatologíadel Hospital Universitario Miguel Servetenlaactualidad. Nuevos retos. Revista española de pediatría: clínica e investigación. 2015; 71:356-62.

14. Jimenez LM, Ramon ML, Casas AA, Arias DP. La Unidad de Cardiología Pediátrica del Hospital Infantil Universitario Miguel Servet de Zaragoza. RevEspPediatr. 2015;71(6):326-31.

15. Mártinez JLV, et al. La Unidad de Cuidados Intensivos Pediátricos enel Hospital Universitario Ramón y Cajalenelaño 2015. RevEspPediatr. 2016;72(2):84-9.

16. Warneck $G$, et al., editors. Annals of intensive care. A multinational survey on the infrastructural quality of paediatric intensive care units.; 2018.105 p. v. 8. 17. Fraisse $A$, Le Bel $S$, Mas B, Macrae D. Paediatric cardiac intensive care unit: current setting and organization in 2010. Arch Cardiovasc Dis. 2010;103(10):546-51.

18. Fry M. Literature review of the impact of nurse practitioners in critical care services. NursCritCare. 2011;16(2):58-66.

19. Zuliani LL, Jericó MC. Estudo comparativo do consumo e gasto com medicamentos em Unidades Pediátricas de Terapia Intensiva e Semi-intensiva. Rev. paul. pediatr. 2012;30:107-15.

20. Moreno JIC, Albert BI, Boni L. Las Unidades de Cardiología Pediátrica y CirugíaCardiaca Infantil del Hospital Universitario y Politécnico La Fe. Rev EspPediatr. 2014;70(2):87-92.

21. Toole CA, et al. Informing Leadership Models: Nursing and Organizational Characteristics of Neonatal Intensive Care Units in Freestanding Children's Hospitals. DimensCrit Care Nurs. 2018;37(3):156-66.

22. Siqueira CL. Competências Gerenciais do Enfermeiro: Teia de competências: conhecimentos, habilidades e atitudes gerenciais para enfermeiros Responsáveis Técnicos Da teoria à prática dos serviços de saúde [Internet]. Pontifícia Universidade Católica de Minas Gerais; 2016 [cited 2019 Jun 6]. Availablefrom: https://www.pucpcaldas.br/uploads/143/manual_competencias_gerenciais_d.pdf

23. Portella AR, et al. Responsabilidade socioambiental por meio da missão, visão e valores: um estudo nas 100 maiores empresas de Santa Catarina. Revista Gestão \& Sustentabilidade Ambiental. 2015:4(1):217-41.

24. Fugulin FMT, Gainzinski RR, Kurcgant P. Sistema de classificação de pacientes: identificação do perfil assistencial dos pacientes das unidades de internação do HU-USP. Rev Latino-am Enfermagem. 2005;13(1):72-8.

25. Da Silva Pinto E, et al., editors. Anais do Salão Internacional de Ensino, Pesquisa e Extensão. Organização do cuidado e trabalho multiprofissional em uti neonatal; 2019. 2019. v. 10.

26. De Oliveira WT, et al. Capacitação de enfermeiros de um hospital universitário público na gestão de custo. Revista de Enfermagem da UFSM. 2014;4(3):566-74.

27. Ministério da Saúde (BR). Portaria $n^{\circ} 1.683$, de 12 de julho de 2007. Aprova, na forma do Anexo, a Norma de Orientação para a Implantação do Método Canguru. Diário Oficial da União. 13 de julho de 2007. Seção 1:4. Disponível em: http://bvsms.saude.gov.br/bvs/saudelegis/gm/2007/ prt1683 12 07 2007.html

28. Galhardi NM, Escobar EMA. Indicadores de qualidade de enfermagem. Revista de Ciências Médicas. 2016:24(2):75-83.

29. Chen $L$, et al. Using the Delphi method to develop nursing-sensitive quality indicators for the NICU. Journalofclinicalnursing. 2017;26(3-4):502-13.

30. Gaíva MAM, Rondon JN, de Jesus LN. Segurança do paciente em unidade de terapia intensiva neonatal: percepção da equipe de enfermagem. Rev. Soc. Bras. Enferm. Ped. 2017:17(1):14-20.

31. Sales $C B$, et al. Protocolos Operacionais Padrão na prática profissional da enfermagem: utilização, fragilidades e potencialidades. Revista Brasileira de Enfermagem. 2018;71(1):126-34. 